
Physics

\title{
Solvable Systems Featuring 2 Dependent Variables Evolving in Discrete-Time via 2 Nonlinearly-Coupled First-Order Recursion Relations with Polynomial Right-Hand Sides
}

Francesco Calogero, Farrin Payandeh

To cite this article: Francesco Calogero, Farrin Payandeh (2019) Solvable Systems Featuring 2 Dependent Variables Evolving in Discrete-Time via 2 Nonlinearly-Coupled First-Order Recursion Relations with Polynomial Right-Hand Sides, Journal of Nonlinear Mathematical Physics 26:2, 273-280, DOI:

https://doi.org/10.1080/14029251.2019.1591729

To link to this article: https://doi.org/10.1080/14029251.2019.1591729

Published online: 04 January 2021 


\title{
Solvable Systems Featuring 2 Dependent Variables Evolving in Discrete-Time via 2 Nonlinearly-Coupled First-Order Recursion Relations with Polynomial Right-Hand Sides
}

\author{
Francesco Calogero $^{a, b, 1}$ and Farrin Payandeh ${ }^{a, c, 2}$ \\ a Physics Department, University of Rome “La Sapienza”, Rome, Italy \\ ${ }^{b}$ INFN, Sezione di Roma 1 \\ ${ }^{c}$ Department of Physics, Payame Noor University (PNU), PO BOX, 19395-3697 Tehran, Iran \\ ${ }^{1}$ francesco.calogero@roma1.infn.it,francesco.calogero@uniromal.it \\ ${ }^{2}$ fpayandeh@pnu.ac.ir,farrinpayandeh@yahoo.com
}

Received 21 November 2018

Accepted 16 December 2018

The evolution equations mentioned in the title of this paper read as follows:

$$
\tilde{x}_{n}=P^{(n)}\left(x_{1}, x_{2}\right), \quad n=1,2,
$$

where $\ell$ is the "discrete-time" independent variable taking integer values $(\ell=0,1,2, \ldots), x_{n} \equiv x_{n}(\ell)$ are the 2 dependent variables, $\tilde{x}_{n} \equiv x_{n}(\ell+1)$, and the 2 functions $P^{(n)}\left(x_{1}, x_{2}\right), n=1,2$, are 2 polynomials in the 2 dependent variables $x_{1}(\ell)$ and $x_{2}(\ell)$. The results reported in this paper have been obtained by an appropriate modification of a recently introduced technique to obtain analogous results in continuous-time $t$-in which case $x_{n} \equiv x_{n}(t)$ and the above recursion relations are replaced by first-order ODEs. Their potential interest is due to the relevance of this kind of evolution equations in various applicative contexts.

\section{Introduction}

In this introductory Section 1, after providing some notational prescriptions, we tersely review previous relevant findings.

Notation 1.1. Hereafter $\ell=0,1,2, \ldots$ denotes the discrete-time independent variable; the dependent variables are $x_{n} \equiv x_{n}(\ell)$ (generally with $n=1,2$ ), and the notation $\tilde{x}_{n} \equiv x_{n}(\ell+1)$ indicates the once-updated values of these variables. We shall also use other dependent variables, for instance $y_{m} \equiv y_{m}(\ell)$, and then of course likewise $\tilde{y}_{m} \equiv y_{m}(\ell+1)$. All variables such as $x, y, z$ (generally equipped with indices) are assumed to be complex numbers, unless otherwise indicated; it shall generally be clear from the context which of these and other quantities depend on time (as occasionally_but not always-explicitly indicated); parameters such as $a, \alpha, \beta, \gamma, A$, etc. (often equipped with indices) are generally time-independent complex numbers; and indices such as $n, m$, $j$ are generally positive integers (the values they may take shall be explicitly indicated or quite clear from the context).

Remark 1.1. In this paper the term solvable generally characterizes systems of evolution equations the initial-values problems of which are explicitly solvable by algebraic operations.

In the following Subsection 1.1 we tersely review-mainly via quotations (with minor adjustments) from a recent paper of ours [1] - a recent approach to identify solvable dynamical systems in continuous-time $t$, as introduction to the extension of (some of) these results to the case of discretetime $\ell$, which is the topic of the present paper. Previous results on solvable discrete-time models 
are tersely reviewed in the subsequent Subsection 1.2. Our main findings are reported in Section 2 (also based on the results reported in Appendix A). A concluding Section 3 outlines tersely possible additional developments.

\subsection{Review of an analogous approach in the continuous-time context}

"Long time ago the idea has been introduced to identify dynamical systems (evolving in continuoustime $t$ ) which are solvable by using as a tool the relations between the time evolutions of the coeffcients and the zeros of a generic time-dependent polynomial [2]. The basic idea of this approach is to relate the time-evolution of the $N$ zeros $x_{n}(t)$ of a generic time-dependent polynomial $p_{N}(z ; t)$ of degree $N$ in its argument $z$,

$$
p_{N}(z ; t)=z^{N}+\sum_{m=1}^{N}\left[y_{m}(t) z^{N-m}\right]=\prod_{n=1}^{N}\left[z-x_{n}(t)\right],
$$

to the time-evolution of its $N$ coefficients $y_{m}(t)$. Indeed, if the time evolution of the $N$ coefficients $y_{m}(t)$ is determined by a system of ODEs which is itself solvable, then the corresponding timeevolution of the $N$ zeros $x_{n}(t)$ is also solvable, via the following 3 steps: (i) given the initial values $x_{n}(0)$, the corresponding initial values $y_{m}(0)$ can be obtained from the explicit formulas-expressing the $N$ coefficients $y_{m}(t)$ of the polynomial (1.1a) in terms of its $N$ zeros $x_{n}(t)$-reading (for all time, hence in particular at $t=0$ )

$$
y_{m}(t)=(-1)^{m} \sum_{1 \leq n_{1}<n_{2}<\cdots<n_{m} \leq N}^{N}\left\{\prod_{\ell=1}^{M}\left[x_{n_{\ell}}(t)\right]\right\}, \quad m=1,2, \ldots, N ;
$$

(ii) from the $N$ values $y_{m}(0)$ thereby obtained, the $N$ values $y_{m}(t)$ are then evaluated via theassumedly solvable - system of ODEs satisfied by the $N$ coefficients $y_{m}(t)$; (iii) the $N$ values $x_{n}(t)$ i.e., the $N$ solutions of the dynamical system satisfied by the $N$ variables $x_{n}(t)$-are then determined as the $N$ zeros of the polynomial, see (1.1a), itself known at time $t$ in terms of its $N$ coefficients $y_{m}(t)$ (the computation of the zeros of a known polynomial being an algebraic operation; of course generally explicitly performable only for polynomials of degree $N \leq 4) \ldots$

The viability of this technique to identify solvable dynamical systems depends of course on the availability of an explicit method to relate the time-evolution of the $N$ zeros of a polynomial to the corresponding time-evolution of its $N$ coefficients. Such a method was indeed provided in [2], opening the way to the identification of a vast class of algebraically solvable dynamical systems (see also, for instance, [3] and references therein); but that approach was essentially restricted to the consideration of linear time evolutions of the coefficients $y_{m}(t)$.

A development allowing to lift this quite strong restriction emerged relatively recently [4], by noticing the validity of the identity

$$
\dot{x}_{n}=-\left[\prod_{\ell=1, \ell \neq n}^{N}\left(x_{n}-x_{\ell}\right)\right]^{-1} \sum_{m=1}^{N}\left[\dot{y}_{m}\left(x_{n}\right)^{N-m}\right]
$$

which provides a convenient explicit relationship among the time evolutions of the $N$ zeros $x_{n}(t)$ and the $N$ coefficients $y_{m}(t)$ of the generic polynomial (1.1a). This allowed a major enlargement of the class of algebraically solvable dynamical systems identifiable via this approach: for many examples see [5] and references therein... 
A new twist of this approach was then provided by its extension to nongeneric polynomials featuring - for all time-multiple zeros. The first step in this direction focussed on time-dependent polynomials featuring for all time a single double zero [6]; and subsequently significant progress has been made to treat the case of polynomials featuring a single zero of arbitrary multiplicity [7]. A convenient method was then provided which is suitable to treat the most general case of polynomials featuring an arbitrary number of zeros each of which features an arbitrary multiplicity. While all these developments might appear to mimic scholastic exercises analogous to the discussion among medieval scholars of how many angels might dance simultaneously on the tip of a needle, they do indeed provide new tools to identify new dynamical systems featuring interesting time evolutions (including systems displaying remarkable behaviors such as isochrony or asymptotic isochrony: see for instance [6] [7]); dynamical systems which—besides their intrinsic mathematical interest—are quite likely to play significant roles in applicative contexts...

We then focused on another twist of this approach to identify new solvable dynamical systems which was introduced quite recently [8]. It is again based on the relations among the time-evolution of the coefficients and the zeros of time-dependent polynomials [4] [5] with multiple roots (see [6], [7] and above); restricting moreover attention to such polynomials featuring only 2 zeros. Again, this might seem such a strong limitation to justify the doubt that the results thereby obtained be of much interest. But the effect of this restriction is to open the possibility to identify algebraically solvable dynamical models characterized by the following systems of 2 ODEs,

$$
\dot{x}_{n}=P^{(n)}\left(x_{1}, x_{2}\right), \quad n=1,2,
$$

with $P^{(n)}\left(x_{1}, x_{2}\right) 2$ polynomials in the 2 dependent variables $x_{1}(t)$ and $x_{2}(t)$; hence systems of considerable interest, both from a theoretical and an applicative point of view (see [8] and references quoted there)." [1]

This completes our review—via a long quotation from a previous paper-of recent developments concerning certain classes of standard dynamical systems in continuous time. In the present paper-after tersely reviewing, in the following Subsection 1.2, some past results in the discretetime context-we focus on the derivation in such a context of analogous results to some of those reported in the continuous-time context in [1].

\subsection{Review of somewhat analogous past findings in the discrete-time context}

Somewhat analogous results to those reviewed in the first part of the previous Subsection 1.1 have been developed over time in the context of discrete-time evolutions, by focussing on the evolution of the zeros of generic monic polynomials the coefficients of which evolve in a solvable manner in discrete time.

The new results reported below consists essential of extensions to the discrete-time context of the results outlined in the second part of the preceding Subsection 1.1. Note however that here and below we actually dispense from a general discussion of the evolution of the zeros of a polynomial the coefficients of which evolve in discrete time in a solvable manner, both in the case of generic monic polynomials (as treated in Chapter 7 of [5]) and in the case of the special polynomials of higher degree than 2 which nevertheless feature for all time only 2 (of course multiple) zeros (as treated in [8], [1]); below we rather employ the simpler technique-described in the following Section 2-to identify solvable nonlinear evolution equations that emerged from that approach and which actually subtends most of the explicit findings reported in [1]. Hence from the previous 
findings for discrete-time evolutions — see [9] and Chapter 7 ("Discrete time") of [5]—we only use below the following discrete-time equivalent of the identity (1.2) (originating from the polynomial (1.1) with $t$ replaced by $\ell$ ),

$$
\prod_{j=1}^{N}\left(\tilde{x}_{n}-x_{j}\right)+\sum_{m=1}^{N}\left[\left(\tilde{y}_{m}-y_{m}\right)\left(\tilde{x}_{n}\right)^{N-m}\right]=0
$$

hence, for the $N=2$ case,

$$
\left(\tilde{x}_{n}-x_{1}\right)\left(\tilde{x}_{n}-x_{2}\right)+\left(\tilde{y}_{1}-y_{1}\right) \tilde{x}_{n}+\tilde{y}_{2}-y_{2}=0, \quad n=1,2
$$

of course with (see (1.1b))

$$
y_{1}(\ell)=-\left[x_{1}(\ell)+x_{2}(\ell)\right], \quad y_{2}(\ell)=x_{1}(\ell) x_{2}(\ell) .
$$

\section{A solvable system of 2 nonlinearly coupled evolution equations in discrete-time satisfied by 2 dependent variables}

In this Section 2 we present our main results, consisting in the identification of a solvable systems of 2 nonlinearly-coupled discrete-time evolution equations belonging to the class

$$
\tilde{x}_{n}=P^{(n)}\left(x_{1}, x_{2}\right), \quad n=1,2,
$$

where the 2 functions $P^{(n)}\left(x_{1}, x_{2}\right)$ are 2 appropriately identified polynomials in the variables $x_{1}(\ell)$ and $x_{2}(\ell)$; a system which is clearly the natural generalization to discrete time of the continuoustime system (1.3). In particular we demonstrate the solvable character of the following dynamical system:

$$
\tilde{z}_{n}=a_{n 1}\left(z_{1}\right)^{2}+a_{n 2}\left(z_{2}\right)^{2}+a_{n 3} z_{1} z_{2}, \quad n=1,2,
$$

with the 6 parameters $a_{n j}(n=1,2, j=1,2,3)$ explicitly given by 6 algebraic expressions in terms of 6 arbitrary parameters.

Let the 2 dependent variables $y_{1}(\ell)$ and $y_{2}(\ell)$ evolve in discrete time according to the following discrete-time evolution equations (the solvability of which is demonstrated in Appendix A):

$$
\tilde{y}_{1}=\alpha\left(y_{1}\right)^{2}, \quad \tilde{y}_{2}=\beta^{2}\left(y_{1}\right)^{2} y_{2}+\gamma\left(y_{1}\right)^{4} ;
$$

and assume again that the 2 variables $y_{1}(\ell)$ and $y_{2}(\ell)$ are related to the 2 variables $x_{1}(\ell)$ and $x_{2}(\ell)$ as follows (see (1.5b)):

$$
y_{1}(\ell)=-\left[x_{1}(\ell)+x_{2}(\ell)\right], \quad y_{2}(\ell)=x_{1}(\ell) x_{2}(\ell) .
$$

Remark 2.1. Let us re-emphasize that, if the discrete-time evolution of the 2 variables $y_{1}(\ell)$ and $y_{2}(\ell)$ is solvable, then the discrete-time evolution of the 2 variables $x_{1}(\ell)$ and $x_{2}(\ell)$ is as well solvable, because the ansatz (2.9) can be inverted via an algebraic operation, indeed quite explicitly, since it clearly implies that $x_{1}(\ell)$ and $x_{2}(\ell)$ are the 2 roots of the following monic polynomial of degree 2:

$$
p_{2}(z)=z^{2}+y_{1} z+y_{2}=\left(z-x_{1}\right)\left(z-x_{2}\right) .
$$

It is then a matter of trivial algebra-either via the formulas (1.5) or directly from (2.8) and (2.9) - to derive the following system of two discrete-time evolution equations satisfied by the 2 
dependent variables $x_{1}(\ell)$ and $x_{2}(\ell)$ :

$$
\left(\tilde{x}_{n}\right)^{2}+\alpha\left(x_{1}+x_{2}\right)^{2} \tilde{x}_{n}+\left(x_{1}+x_{2}\right)^{2}\left[\beta^{2} x_{1} x_{2}+\gamma\left(x_{1}+x_{2}\right)^{2}\right]=0,
$$

implying

$$
\tilde{x}_{n}=-\frac{\alpha\left(x_{1}+x_{2}\right)^{2}+(-1)^{n} \Delta}{2},
$$

with

$$
\Delta^{2}=\left(x_{1}+x_{2}\right)^{2}\left[\left(\alpha^{2}-4 \gamma\right)\left(x_{1}+x_{2}\right)^{2}-4 \beta^{2} x_{1} x_{2}\right] .
$$

Assume now that

$$
\gamma=\frac{\alpha^{2}-\beta^{2}}{4}
$$

so that the right-hand side of (2.10c) become an exact square implying

$$
\Delta= \pm \beta\left[\left(x_{1}\right)^{2}-\left(x_{2}\right)^{2}\right] .
$$

Then clearly

$$
\tilde{x}_{n}=-\frac{\alpha\left(x_{1}+x_{2}\right)^{2}+(-1)^{n} \beta\left[\left(x_{1}\right)^{2}-\left(x_{2}\right)^{2}\right]}{2}, \quad n=1,2,
$$

namely

$$
\tilde{x}_{n}=a_{1}^{(n)}\left(x_{1}\right)^{2}+a_{2}^{(n)}\left(x_{2}\right)^{2}+a_{3}^{(n)} x_{1} x_{2}, \quad n=1,2,
$$

with

$$
a_{1}^{(n)}=-\frac{\alpha+(-1)^{n} \beta}{2}, \quad a_{2}^{(n)}=-\frac{\alpha+(-1)^{n} \beta}{2}, \quad a_{3}^{(n)}=-\alpha
$$

We have thereby identified a simple solvable system of type (2.6), featuring in its right-hand side 2 homogeneous polynomials of second degree in the 2 variables $x_{1}(\ell)$ and $x_{2}(\ell)$, the 6 coefficients $a_{j}^{(n)}(n=1,2, j=1,2,3)$ of which depend on the 2 a priori arbitrary parameters $\alpha$ and $\beta$, see $(2.12 \mathrm{c})$.

From this system an analogous system featuring more arbitrary parameters can be identified via the simple trick of introducing 2 new dependent variables, $z_{1}(\ell)$ and $z_{2}(\ell)$, related linearly to the 2 variables $x_{1}(\ell)$ and $x_{2}(\ell)$ :

$$
\begin{gathered}
z_{1}=A_{11} x_{1}+A_{12} x_{2}, \quad z_{2}=A_{21} x_{1}+A_{22} x_{2}, \\
x_{1}=\left(A_{22} z_{1}-A_{12} z_{2}\right) / D, \quad x_{2}=\left(-A_{21} z_{1}+A_{11} z_{2}\right) / D, \\
D=A_{11} A_{22}-A_{12} A_{21} .
\end{gathered}
$$

It is easily seen that the new system is then just the system (2.7), with the 6 parameters $a_{n j}(n=1,2$, $j=1,2,3)$ explicitly expressed as follows in terms of the 4 arbitrary parameters $A_{n m}(n=1,2$, 
$m=1,2)$ and the 2 arbitrary parameters $\alpha$ and $\beta$ (see (2.12c)):

$$
\begin{aligned}
a_{n 1} & =D^{-2}\left[\left(A_{22}\right)^{2}\left(A_{n 1} a_{1}^{(1)}+A_{n 2} a_{1}^{(2)}\right)+\left(A_{21}\right)^{2}\left(A_{n 1} a_{2}^{(1)}+A_{n 2} a_{2}^{(2)}\right)\right. \\
& \left.-A_{22} A_{21}\left(A_{n 1} a_{3}^{(1)}+A_{n 2} a_{3}^{(2)}\right)\right], \quad n=1,2, \\
a_{n 2}= & D^{-2}\left[\left(A_{12}\right)^{2}\left(A_{n 1} a_{1}^{(1)}+A_{n 2} a_{1}^{(2)}\right)+\left(A_{11}\right)^{2}\left(A_{n 1} a_{2}^{(1)}+A_{n 2} a_{2}^{(2)}\right)\right. \\
& \left.-A_{11} A_{12}\left(A_{n 1} a_{3}^{(1)}+A_{n 2} a_{3}^{(2)}\right)\right], \quad n=1,2, \\
& \\
a_{n 3}= & D^{-2}\left[-2 A_{12} A_{22}\left(A_{n 1} a_{1}^{(1)}+A_{n 2} a_{1}^{(2)}\right)-2 A_{21} A_{11}\left(A_{n 1} a_{2}^{(1)}+A_{n 2} a_{2}^{(2)}\right)\right. \\
+ & \left.\left(A_{11} A_{22}+A_{12} A_{21}\right)\left(A_{n 1} a_{3}^{(1)}+A_{n 2} a_{3}^{(2)}\right)\right], \quad n=1,2 .
\end{aligned}
$$

For the inversion of these transformations-i.e., the issue of expressing the 4 parameters $A_{n m}$ $(n=1,2 ; m=1,2)$ and the 2 parameters $\alpha, \beta$ (see (2.12c)) in terms of the 6 parameters $a_{n \ell}(n=1,2$, $\ell=1,2,3)$-we refer to the analogous discussion in [1].

\section{Outlook}

In this final Section 3 we outline tersely possible future developments of the findings reported above.

The findings reported in this paper extend to evolutions in discrete-time only some of the findings for evolutions in continuous-time reported in [8] and [1] and tersely reviewed above (in Section 1). It is therefore quite natural to envisage an extension from the continuous-time context to the discrete-time context of other results reported in [8] and [1].

A different research line might be directed towards applications of the solvable discrete-time evolution equation (2.7), including its generalization via an assigned shift of the dependent variables $z_{n}(\ell)$, say

$$
z_{n}(\ell)=w_{n}(\ell)+f_{n}(\ell), \quad n=1,2,
$$

with $f_{n}(\ell)$ two arbitrarily assigned functions of the discrete-time $\ell$, implying that the new dependent-variable $w_{n}(\ell)$ satisfies the (of course still solvable) discrete-time evolution equation

$$
\begin{aligned}
\tilde{w}_{n} & =a_{n 1}\left(w_{1}\right)^{2}+a_{n 2}\left(w_{2}\right)^{2}+a_{n 3} w_{1} w_{2}+g_{n 1} w_{1}+g_{n 2} w_{2}+h_{n}, \\
g_{n 1}(\ell) & \equiv 2 a_{n 1} f_{1}(\ell)+a_{n 3} f_{2}(\ell), \quad g_{n 2}(\ell) \equiv 2 a_{n 2} f_{2}(\ell)+a_{n 3} f_{1}(\ell), \\
h_{n}(\ell) & \equiv a_{n 1}\left[f_{1}(\ell)\right]^{2}+a_{n 2}\left[f_{2}(\ell)\right]^{2}+a_{n 3} f_{1}(\ell) f_{2}(\ell)-f_{n}(\ell+1), \\
n & =1,2 .
\end{aligned}
$$

\section{Acknowledgements}

FP likes to thank the Physics Department of the University of Rome "La Sapienza" for the hospitality from April to November 2018 (during her sabbatical), when the results reported in this paper were obtained. 


\section{F. Calogero and F. Payandeh / Solvable Systems Featuring 2 Dependent Variables...}

\section{Appendix A. A useful class of solvable systems of 2 nonlinear discrete-time evolution equations for the 2 variables $y_{m}(\ell)$}

The system (2.8) of discrete-time evolution equations discussed in this Appendix A is too simple to justify considering its solution as a new finding; its solution is reported here because of its role in solving the novel, more interesting, discrete-time model discussed above. The solution of the initial-value problem of the first of the 2 discrete-time evolution equations (2.8),

$$
\tilde{y}_{1}=\alpha\left(y_{1}\right)^{2}
$$

is an easy task:

$$
y_{1}(\ell)=\alpha^{-1}\left[\alpha y_{1}(0)\right]^{2^{\ell}}, \quad \ell=0,1,2, \ldots
$$

To solve the initial-value problem for the second of the 2 discrete-time evolution equations (2.8),

$$
\tilde{y}_{2}=\beta^{2}\left(y_{1}\right)^{2} y_{2}+\gamma\left(y_{1}\right)^{4},
$$

it is convenient to introduce the ansatz

$$
y_{2}(\ell)=\left\{\beta^{2 \ell} \prod_{s=0}^{\ell-1}\left[y_{1}(s)\right]^{2}\right\} Y(\ell),
$$

implying

$$
Y(0)=y_{2}(0)
$$

Remark A.1. We always use the standard convention according to which, if $s_{1}>s_{2}$,

$$
\sum_{s=s_{1}}^{s_{2}} f(s)=0, \quad \prod_{s=s_{1}}^{s_{2}} f(s)=1
$$

for any arbitrary function $f(s)$ of the discrete-time variable $s$.

It is then easily seen that (A.2) implies

$$
Y(\ell+1)=Y(\ell)+F(\ell)
$$

with

$$
F(\ell)=\gamma\left[y_{1}(\ell)\right]^{4} \beta^{-2(\ell+1)} \prod_{s=0}^{\ell}\left[y_{1}(s)\right]^{-2},
$$

implying, via (A.1b),

$$
F(\ell)=\gamma \alpha^{-4}(\alpha / \beta)^{2(\ell+1)}\left[\alpha y_{1}(0)\right]^{2} .
$$

Hence in conclusion, from (A.5) with (A.3b),

$$
Y(\ell)=y_{2}(0)+\gamma(\alpha \beta)^{-2}\left[\frac{(\alpha / \beta)^{2 \ell}-1}{(\alpha / \beta)^{2}-1}\right]\left[\alpha y_{1}(0)\right]^{2},
$$


hence, via (A.3a),

$$
\begin{aligned}
& y_{2}(\ell)=(\beta / \alpha)^{2 \ell}\left[\alpha y_{1}(0)\right]^{2^{\ell+1}-2}\left\{y_{2}(0)\right. \\
& \left.+\gamma(\alpha \beta)^{-2}\left[\frac{(\alpha / \beta)^{2 \ell}-1}{(\alpha / \beta)^{2}-1}\right]\left[\alpha y_{1}(0)\right]^{2}\right\} .
\end{aligned}
$$

The 2 formulas (A.1b) and (A.7) provide the explicit solution of the initial-values problem of the discrete-time evolution (2.8). Of course in (A.1b), to make this solution applicable to the final findings reported in Section 2, the assignment $\gamma=\left(\alpha^{2}-\beta^{2}\right) / 4$ must be made, see (2.11a).

\section{References}

[1] F. Calogero and F. Payandeh, "Polynomials with multiple zeros and solvable dynamical systems including models in the plane with polynomial interactions", J. Math. Phys. (submitted to, 20.11.2018).

[2] F. Calogero, "Motion of Poles and Zeros of Special Solutions of Nonlinear and Linear Partial Differential Equations, and Related "Solvable" Many-Body Problems", Nuovo Cimento 43B, 177-241 (1978).

[3] F. Calogero, Classical many-body problems amenable to exact treatments, Lecture Notes in Physics Monograph m66, Springer, Heidelberg, 2001 (749 pages).

[4] F. Calogero, "New solvable variants of the goldfish many-body problem", Studies Appl. Math. 137 (1), 123-139 (2016); DOI: 10.1111/sapm.12096.

[5] F. Calogero, Zeros of Polynomials and Solvable Nonlinear Evolution Equations, Cambridge University Press, Cambridge, U.K., 2018 (168 pages).

[6] O. Bihun and F. Calogero, "Time-dependent polynomials with one double root, and related new solvable systems of nonlinear evolution equations", Qual. Theory Dyn. Syst. (published online: 26 July 2018). doi.org/10.1007/s12346-018-0282-3; http://arxiv.org/abs/1806.07502.

[7] O. Bihun, "Time-dependent polynomials with one multiple root and new solvable dynamical systems", arXiv:1808.00512v1 [math-ph] 1 Aug 2018.

[8] F. Calogero and F. Payandeh, "Solvable dynamical systems in the plane with polynomial interactions", to be published as a chapter in a collective book to celebrate the 65th birthdate of Emma Previato (in press).

[9] O. Bihun and F. Calogero, "Generations of solvable discrete-time dynamical systems", J. Math. Phys. 58, 052701 (2017); DOI: 10.1063/1.4928959. 